\title{
47 \\ Cooperative Design on the Internet
}

\author{
L. Nemes ${ }^{1}$, P. Bertok ${ }^{2}$ \\ ${ }^{1}$ Division of Manufacturing Technology, \\ Commonwealth Scientific and Industrial Research Organisation, \\ Locked Bag No. 9, Preston 3072, Australia \\ Phone: 6139662 7707, Fax: 6136627851 \\ email:lnm@mlb.dmt.csiro.au \\ ${ }^{2}$ Department of Computer Science, \\ Royal Melbourne Institute of Technology \\ PO Box 71, Bundoora 3083, Australia \\ Phone: 6139468 b 2709, Fax: 613946789708 \\ email:pbertok@rmit.edu.au
}

\begin{abstract}
A new type of cooperative engineering has appeared on the horizon. Notwithstanding geographical separation designers can share design data and model, cooperate through interactions on-line. In this paper the major issues of this method are examined, together with its impact on the design process. An overview of several typical cooperative design systems is also given.
\end{abstract}

\section{Keywords}

Computer Aided Design, Internet, Computer Supported Cooperative Work

\section{INTRODUCTION}

Computer supported cooperative work $(\mathrm{CSCW})$ is a relatively new field, but its increasing popularity clearly indicates a demand for this kind of tools. The range of applications is really wide, as many professional activities include cooperative efforts. The first question is, however, whether the technology is mature enough to enable real life application of CSCW systems, or it is still in the experimental stage. The second, almost equally important issue is, the method of collaboration between users separated by hundreds or thousands of kilometres, and whether any public network can be used as an efficient communication medium for collaborative work. In this paper we are going to focus on a special application, cooperative engineering design, and examine the current state of the art. Trends will also be analysed and future implementations will be envisaged. 


\section{THE DESIGN ENVIRONMENT}

\section{The (engineering) design process}

Design engineers spend a great deal of time with gathering and organising information, negotiating trade-offs with other co-designers or users, etc., or producing new ideas in a collective manner. Despite the cooperative nature of the design process, traditional CAD systems provide minimum support, if at all, in this area, and face-to-face interaction is the usual way to solve problems. When collaborating designers are at geographically distant locations, sufficiently regular face-to-face meetings are not feasible.

$\mathrm{CSCW}$ tools and concurrent engineering have been in the centre of attention for several years, but most designers are still exchanging information off-line via email messages, or are manually downloading files. In truly cooperative systems on-line information sharing is desired, where details and logs of solutions, changes, annotations are accessible for each member of the design group. This is especially important when the group members are scattered over a wide geographical area. The use of public data networks for communication between group members can be an obvious choice. The pervasiveness of the Internet and its cost effectiveness makes it very attractive for this kind of applications. In particular, new applications on the Internet, such as the WorldWide Web, can offer new perspective for using the Internet for CSCW in design.

\section{Engineering design and the World-wide Web $(W W W)$}

There have been several proposals to involve the WWW in engineering design, and they include the WWW to different extent. The first step is browsing among information pages (eg. component data) published by suppliers on the Web. In this case the WWW is used only as a storage of information, as an on-line catalogue.

The next step is when services offered through the Web perform some operations on the users' data, eg. testing or verifying a certain design. In this case the Web still serves only as a communication tool transferring data from one site to another. The most advanced stage is, when the services offered by the Web are fully exploited by using information servers, that are part of the design system, over the network.

Interactions between co-designers are of vital importance. They include not only design data exchange/modification, but also information, opinion about individual design solutions, as well as ideas, suggestions. The information can be shared off-line or online, as shown in Figure 1. These interactions can be synchronous (also called concurrent), as in a conversation, or asynchronous, as in a mail exchange. Synchronous interactions are sensitive to latency caused by network delays and session control, and the performance of the Internet/WWW has to be examined from this aspect.

Access control and data security pose a difficult question. There are established methods for handling concurrent access in distributed systems, but access rights can be difficult to handle in a system with communication channels as exposed as the Internet is. For example user A may want to share a set of objects with user B, another set of objects with user $\mathrm{C}$, and may also have private objects not to be shared with anyone. Access rights can also change, eg. when a designer needs the approval of a co-designer, access has to be provided to relevant data, that was private beforehand. 


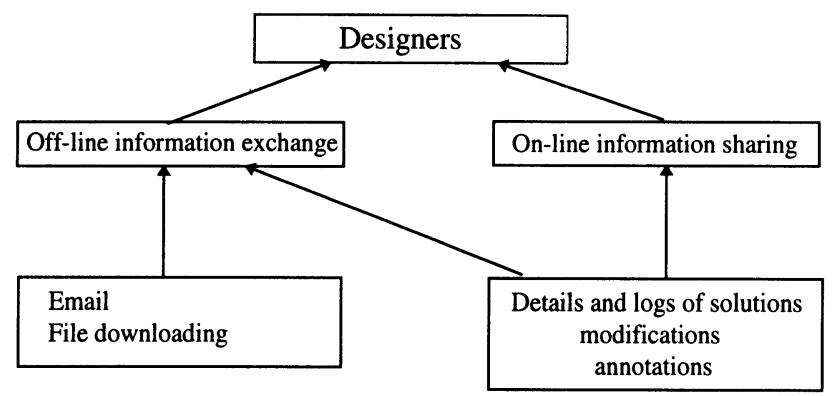

Figure 1. Information sharing between designers.

In traditional environment a design system is unique to the application. Its framework and data structure is specific to that system, and this severely restricts the cooperation between different design teams, but it is still not enough to provide security, eg. against stealing a design, even locally. Moving data between different sites aggravates the problem to a great extent.

The first security problem is the protection of data objects and the intellectual property it may represent, when it is transferred through the open channels of the Internet. Tempering with data is less likely, but eavesdropping or obtaining data inadvertently is a very real danger. The second, and potentially greater problem is downloading data to remote sites for further processing, eg. for testing by a subcontractor. As the remote site can interpret the data, very strict access control has to be provided at that site as well.

It has to be mentioned that many of these systems were developed in an evolutionary manner, ie. by adding newer modules to existing systems. However, to take full advantage of CSCW and Internet based services a systematic approach is needed when building such systems.

\section{COOPERATIVE DESIGN SYSTEMS USING THE INTERNET}

\subsection{The Henry system}

The Henry VLSI design system tries to integrate heterogeneous data, frameworks and on-line services. It accommodates remote command executions and links between heterogeneous tools. One of the most prominent features of the system is that it integrates a CAD system with commercial services available on the Internet, and downloaded information can become part of the design.

It evolved from a multimedia design system, which used a common front-end to multiple design tools [Silva, 1995]. Links between different systems were described in files, and remote services included data and program execution. It was the openness of the original system that enabled interfacing with Internet based services, such as the WWW. The main advantage of interfacing to the Internet was that (commercial) 
services could be easily utilised, such as searching through on-line catalogues etc. The designers of the Henry system also envisage CAD outsourcing, whereby specialised services, eg. simulation and design verification, will also be commercially available. This would assume, however, general compatibility between different systems. As very widely used software systems are still experiencing difficulties in data compatibility, this application will probably be limited to the cooperation of a few systems in a closed circle.

The core of the Henry system is an integrated design and documentation environment. It uses files with hyperlinks to other tools, which are called active documents, because they can send and receive commands and data. The intertool messages are transferred via the standard email (SMTP) service. In more complex cases, when messages contain non-textual information as well, the HTTP protocol is used. Internet message service protocols constitute also the framework for integration of heterogeneous tools. The system's message servers are using these protocols only, and use Internet addresses. For security reasons messages arrive to the servers through an untrusted interface, and sensitive operations, such as file writing, are performed by a trusted part of the system, which prompts the user before granting access to resources. The interface for communication with external services has two gateway protocols: email and HTTP. Outgoing messages are sent by using standard Unix utilities (sendmail or telnet), and the system is configured to automatically forward related messages to Henry tools. The Henry system does not include security features like encryption or authentication.

\subsection{The SHAREd Web system}

The SHARE project is aimed at Internet based collaborative design. It originally started with an enhanced email service (MIME) transferring messages between sites. An application, built on $\mathrm{X}$ tools and called $\mathrm{X}$ share, was used for sharing applications in a shared environment. The sharing was simple: one application was running multiple display servers, and thus avoided the problems arising from object replication. The cooperative work was based on the feature of the mail service that it could run applications, such as $\mathrm{X}$ share, from within its own environment. This approach allowed sharing data, but collaboration between designers is very limited, and audio and video facilities had to be installed [Glicksman, 1993]. To reduce communication time and costs a common information base was set up, where most of the documents, such as CAD files, were stored, and messages between designers contained only references (pointers) to objects in the information base [Toye 1993].

The WWW offered new and fast communication between cooperating designers. Other standard services could also be easily integrated with system. WWW (HTTP) communication was built into the system together with other utilities, such as HyperMail, that converts email messages to HTML documents. [Kumar, 1994].

The system also supports collaboration efforts by providing tools for interaction between participants. Off-line tools include a multimedia message service that can also handle annotations to a message, so the user can see a document with or without certain modifications. Mail messages can have references to other messages, follow hypertext links and be looked at with WWW browsers. Messages can contain synchronised recordings of mouse motions and audio data, which can be played back at the recipient's 
machine. On-line collaboration is also supported on a basic level. A shared version of Mosaic allows common browsing by providing replicas of the current screen to participants. No concurrency control is built into the system, ie. the first user clicking on a document will get priority. Problems due to concurrent access and possible raceconditions are reduced by the fact that the common browsing sessions are used together with audio/video conferencing. The system had an Internet based multimedia conferencing tool, that offers audio, video connections and a shared whiteboard.

The developers have conducted an experimental design session with the aim of testing the benefits of the implemented services. The most successful service was the shared whiteboard, particularly for tasks that could be solved in two dimensions. The email connection was also useful, but needed a backup fax connection. The network audio and video connections were less successful, and the users of the audio service had to revert to the phone service. The likely cause of the problem was the unpredictable nature of network delays and latency, as they depend on network load and traffic volume. The shared X based applications had mixed results. While cooperation was good between similar machines, format compatibility and conversion problems arose between different machines. This indicates that different low-level $\mathrm{X}$ implementations may not provide unconditional design portability. The participants had to revert to traditional services again, to the fax service in this case, to exchange design information.

\subsection{Knowledge Aided Design (KAD)}

$\mathrm{KAD}$, a system still under development, is designed to support concurrent engineering design and enhance the degree of cooperation among design team members [Leizerowicz, 1996]. It focuses on knowledge management, uses the WWW for coordinating activities, information access and visualisation.

The first coordination tool called Distributed Management System (DMS) and developed on the WWW uses an Oracle RDBMS as a back-end. The main task of DMS is to trace the knowledge exchanged via email messages, categorise, archive and distribute messages. The stored messages then can be accessed as data, and examined/queried by keywords, threads or in chronological order. DMS is independent of the actual design task, and being based on the WWW it provides a universal platform for permanent information storage across different systems.

Another problem addressed by the system developers was information access control. Some information has to be available without restrictions, while access to other data or documents may be very limited. The concepts used here were similar to those used in SQL, but they were implemented through WWW authentication and access control, rather than through database management facilities. The method used was setting up easily configurable masks that control the read and write of individual objects. Templates are defined for different user groups, such as designers, customers, etc., and the template used is determined by the identity of the user, regardless of the user's geographical location.

The system has a WWW interface, and different consistency and error checking functions are implemented through that interface. A very important benefit of WWW (HTML) documents to this application was that they provide instant global access to up 
to date information without any special effort. For instance, reports can be generated about inconsistencies introduced to a design through making some changes by immediately accessing information in a knowledge base. Old designs can also be retrieved by performing parametric and regular expression searches. The WWW interface's ability to handle and display graphical information was used for visualisation of hierarchical and networked information.

\subsection{SEPIA}

SEPIA is an early cooperative system. Even though it did not address many problems, wide area collaboration in particular, it was a very important milestone. It set up a framework for cooperative design, and clearly identified the main points of interest. It also classified the different activities that make up the design process, and provided support for them.

SEPIA is a cooperative hypermedia environment, providing solutions for using hypermedia tools both in single-author or multiple-author environments. Both document reading and preparation need support, although to different extent. Even in a single-user environment readers need help in navigation, ie. in finding the appropriate document, and in the comprehension process, in understanding the overall structure of the document and the semantic links [Streitz, 1992]. Preparing a document or design is even more complex, as first it has to be conceived, together with its attachments or dependent documents, and then translated into a hypermedia document. In a multipleauthor environment document sharing has also to be considered, the work can be individual, loosely coupled or tightly coupled. SEPIA was designed to work in such environment, and support the smooth transition between the different modes of operation. It provides services on two levels: on the upper level it supports navigation among related documents, and on the lower level it enables reading and writing/creating documents. The system works in a multi-window environment, where the user opens additional windows when reaching new documents. One window always shows the structure of the entire document.

The design starts with putting the ideas into a structured hypertext framework. Then the author finalises the document, includes all the necessary details and connections. In the next step the document is prepared for "publishing", ie. additional details, issues for further consideration are appended to it. Up to this point, the document is under the full control of the creator only. Then it is made available to other authors. Cooperative work is performed only in the last phase, when the document is open for "argumentation", and co-authors can add, modify design objects, elaborate arguments for supporting or objecting particular solutions. The cooperation starts in loosely coupled mode, when different users can view the same object at the same time. Commands for displaying an object or changing the view have only local effect, but any changes made on the object itself will immediately be shown on all screens displaying the object. Users can join a loosely coupled session freely, and other users working on the same object are notified when the new user opens a node already used by them. When closer cooperation is needed, the system is switched from loosely coupled to tightly coupled mode. In that mode the users share the same view of the common object. When a tightly coupled session starts, every participant of the loosely coupled session is invited to join, and they are asked to confirm their participation. 


\subsection{World-Wide Web based support for cooperative design}

There are several new tools that can facilitate the use of the WWW for design purposes, although they may not have been specifically developed for this purpose. One of them is Web*, that provides a framework for HTTP servers with dynamically changing data [Almasi, 1995]. It allows WWW based documents to link other files through CGI scripts dynamically. Web* pages can contain variables and tables, in addition to usual HTML information. While traditional HTML documents are interpreted only once at the client side, Web* documents are interpreted twice: first on the server side and then on the client side. In the first step all references to variables etc. are resolved at the server side, and then a standard HTML format document is transferred through the net. This feature facilitates the organisation of documents, and can be particularly useful in describing and documenting a design, or relationships between its components, while it is still evolving. Other systems, like COMO, offer shared graphics screens that operate on the what you see is what I see (WYSIWIS) principle [Gall, 1996].

\section{USES, LIMITATIONS, FUTURE DIRECTIONS}

The cooperative design systems involving the Internet in the design process have indicated several important benefits from globalising the design process. The first advantage is access to various information across the Internet, in addition to data specific to the system. As companies put detailed information about their products and services on the WWW to help current and potential customers, searching on the WWW has emerged and opened the way for Yellow Pages type services. Even purchase orders through the WWW have become common, which may lead to faster prototyping. One system, SHAREd Web, was used as a design aid in the classroom. Its primary function was to contain data about students and supervisors in the classroom projects as well as information on related projects, university and corporate R \& D sites etc. Among the most accessed parts of its data base were the White and Yellow Pages of local business selling components or providing services useful for the projects.

Another new service through the Internet is simultaneous interaction, eg. the transfer of audio and video data for conferencing. At first it seemed that this opens the way for multimedia conferencing, which could be extremely helpful in cooperative design. Unfortunately the experiments performed with this method did not prove to be successful. The main problem was instantaneous availability of bandwidth, as the Internet does not guarantee a maximum data propagation delay. Late arriving or missing packets will cause a distortion in the signal reconstructed at the receiving end. This factor is more important with audio than with video data, as the human ear is more sensitive to signal distortion than the eye. In fact, this problem is not unique to audio or video data, traditional CSCW systems have a similar problem: the screen update time includes the network delays. Although this problem may be alleviated by the proliferation of ATM networks that provide such a service, the cost of using such networks may be prohibitive in many cases. The question is rather, if there is a real need for serialised communication between cooperating partners, such as the well defined turn taking in human dialogue. The computer can store the record of activity the same way as it happens in writing. This allows overlapping or concurrent communication, where participants do not have to wait for their turns to perform an activity. For 
example, two designers can start modifying different parts in the same section of a design, and when completed, first they can exchange the solutions to see what the other did, before merging them into one new design. Automatic merging, however, may not be feasible because of the possible dependencies between components.

Internet tools can also be used for access control, eg. WWW based authentication is used in the KAD system. While this can be a good solution to control regular access to data, the protection provided by this method against eavesdropping or malicious attack is very limited. Additional protection, eg. firewalls and screening of messages may be necessary. Another security related problem is the protection of data at remote sites where the data can be fully interpreted, and the intellectual property data represent can be exposed to unauthorised users, either intentionally or unintentionally. Commonly used Internet security mechanisms do not offer obvious solutions for this problem. It needs additional security policies and mechanisms, in both computer and non-computer related communication.

It is almost certain that $\mathrm{CAD}$ systems developed in the future will support interactions through a local/wide area network. They will exploit new tools, like the Java language, heavily. The services offered by these systems for geographically distributed users may concentrate on the communication between designers and other auxiliary activities, rather than on the design product itself. Many of these services have already matured together with the underlying infrastructure developments, (eg. component ordering). It can be reasonably expected that the extremely fast pace of development in infrastructure will provide new and/or better solutions in many areas.

\begin{tabular}{|ll|}
\hline Expected developments: & $\begin{array}{l}\text { WWW use for design } \\
\text { Improved on-line data exchange }\end{array}$ \\
\hline Improved solutions needed: & $\begin{array}{l}\text { Interactions between designers } \\
\text { Security issues }\end{array}$ \\
\hline New methods needed: & $\begin{array}{l}\text { Multi user support for the design product } \\
\text { data base }\end{array}$ \\
\hline
\end{tabular}

Figure 2. Steps for future directions

\section{CONCLUSION}

The use of the Internet for computer supported cooperative work has significant benefits. The network is public, easily accessible, and can carry large amount of data in a cost efficient way. It also serves as a large information source, as many companies are publishing data about products and services through the World-wide Web.

Some limitations to the use of the Internet for design activities have also become clear. The security and data protection mechanisms provided by the Internet are not sufficient for cooperative design, especially for protecting data at a remote user site. Another, probably even more serious limitation is the lack of ability to transfer synchronous or isochronous data for voice and video communications, and probably new interaction mechanisms will have to be introduced for the purpose. 


\section{REFERENCES}

G. Almasi, A. Suvaiala, I. Muslea, C. Cascaval, T. Davis, V. Jagannathan: Web* - A Technology to Make Information Available on the Web, WETICE '95, the IEEE Fourth Workshop on Enabling Technologies, 1995

U. Gall, J. Kautz: The COMO system, http://www4.informatik.uni-erlangen.de/immdiv/projects/como/, April 1996

J. Glicksman, V. Kumar: A SHAREd Collaborative Environment for Mechanical Engineers, Proc. of $\quad$ Groupware (ftp://ftp.eit.com/pub/share/papers/groupware93.ps)

V. Kumar, J. Glicksman, G.A. Kramer: A SHAREd Web to Support Design Team, Proc. of at WETICE '94, the IEEE Third Workshop on Enabling Technologies, 1996

W. Leizerowicz, T. Bilgic, J. Lin, M. S. Fox: Collaborative Design Using the WWW, Presented at WETICE '96, the IEEE Fifth Workshop on Enabling Technologies, 1996

M.J. Silva, R.H. Katz: The Case for Design Using the World Wide Web, Proc. of the 32nd ACM/IEEE Design Automation Conference, San Francisco, California, 1995

N. Streitz, J. Haake, J. Hannemann, A. Lemke, W. Schuler, H. Schutt, M. Thuring: SEPIA, A Cooperative Hypermedia Authoring Environment, Proc. of the 4th ACM Conference on Hypertext, ECHT '92, Milan, Italy 1992

G. Toye, M. R. Cutkosky, L. J. Leifer, J. M. Tenenbaum, J. Glicksman: SHARE: a Methodology and Environment for Collaborative Product Development, Int. J. of Intelligent and Cooperative Information Systems, in Proc.of Infrastructure for Collaborative Enterprises, IEEE, 1993

\section{BIOGRAPHY}

Laszlo Nemes is an Assistant Chief, and Leader of the Intelligent Manufacturing Systems Program at the Division of Manufacturing Technology, Commonwealth Scientific and Industrial Research Organisation, Australia. He is a member of the Board of Directors, CRC for Intelligent Manufacturing Systems and Technologies. Dr Nemes is a member of the Technical Committee for the international research project on Enterprise Integration for Global Manufacturing (Globeman '21).

His research areas include manufacturing systems, enterprise integration, reference architectures, modelling techniques; man-machine interfaces; concurrent engineering. He has published four monographs, 56 referred journal and conference papers, 27 invited papers and keynote addresses at international meetings. He gave lectures and seminars in the USA, Mexico, Egypt, Iraq and Australia. He co-authored 23 reports and 6 patents. Dr Nemes has been awarded with the Silver Core of IFIP; he is a fellow of the IE Aust. and FISPE USA; member os the MJSPE Japan; chairman of the WG 5.3 of IFIP.

Peter Bertok is lecturing computer science at the Royal Melbourne Institute of technology, Australia. Prior to that, he was a senior computer scientist at a company specialised in simulation software, in Melbourne. He had been a visiting research fellow at the Tokyo University, Japan. Dr Bertok's research interests include distributed computing systems, CSCW architectures, data communications and real time systems. 
He has published a monograph, 34 refereed conference \& journal papers and a patent. $\mathrm{He}$ was an editor of conference proceedings and served on the international paper committee of various conferences. Dr Bertok is a member of IFIP WG 5.3, IEEE Computer Society, IEEE Robotics and Automation Society. 